PROCEEDINGS OF THE

AMERICAN MATHEMATICAL SOCIETY

Volume 129, Number 12, Pages 3453-3460

S 0002-9939(01)06299-2

Article electronically published on June 27, 2001

\title{
EQUIPARTITION OF INTERVAL PARTITIONS AND AN APPLICATION TO NUMBER THEORY
}

\author{
KARMA DAJANI AND ADAM FIELDSTEEL
}

(Communicated by Michael Handel)

\begin{abstract}
We give wider application and simpler proofs of results describing the rate at which the digits of one number-theoretic expansion determine those of another. The proofs are based on general measure-theoretic covering arguments and not on the dynamics of specific maps.
\end{abstract}

\section{INTRODUCTION}

Let $x \in[0,1)$, and suppose we are given the first $n$ digits of some numbertheoretic expansion of $x$ (what such an expansion is will be defined in a moment). Then these $n$ digits determine $m(n, x)$ digits of some other expansion of $x$. In this paper we address the question: what can one say about the limit

$$
\lim _{n \rightarrow \infty} \frac{m(n, x)}{n}
$$

for almost all $x \in[0,1)$ ?

In 1964, G. Lochs [L] was the first to address this question when comparing the decimal and the continued fraction expansions. Let $x=. d_{1} d_{2} \cdots$ be the decimal expansion of $x$ (which is generated by iterating the map $S x=10 x(\bmod 1))$, and let

$$
x=\frac{1}{a_{1}+\frac{1}{a_{2}+\frac{1}{a_{3}+\frac{1}{\ddots}}}}=\left[0 ; a_{1}, a_{2}, \cdots\right]
$$

be its regular continued fraction (RCF) expansion (generated by the map $T x=$ $\left.\frac{1}{x}-\left\lfloor\frac{1}{x}\right\rfloor\right)$. Let $y=d_{1} d_{2} \cdots d_{n}$ be the rational number determined by the first $n$ decimal digits of $x$, and let $z=y+10^{-n}$. Then, $[y, z]$ is the decimal cylinder of order $n$ containing $x$, which we also denote by $B_{n}(x)$. Now let $y=\left[0 ; b_{1}, b_{2}, \cdots, b_{l}\right]$ and $z=\left[0 ; c_{1}, c_{2}, \cdots, c_{k}\right]$ be the RCF expansion of $y$ and $z$. Let

$$
m(n, x)=\max \left\{i \leq \max (l, k): \text { for all } j \leq i, b_{j}=c_{j}\right\} .
$$

Received by the editors March 8, 2000.

1991 Mathematics Subject Classification. Primary 11Kxx, 28Dxx.

Key words and phrases. Entropy, ergodic, continued fraction, number-theoretic fibered map.

The first author was partially supported by NWO grant no. 61-449.

We wish to thank Ethan Coven for useful observations. 
In other words, $m(n, x)$ is the largest integer such that $B_{n}(x) \subset C_{m(n, x)}(x)$, where $C_{j}(x)$ denotes the continued fraction cylinder of order $j$ containing $x$. Lochs $\mathrm{L}$ proved the following theorem:

Theorem 1. Let $\lambda$ denote Lebesgue measure on $[0,1)$. Then for a.e. $x \in[0,1)$

$$
\lim _{n \rightarrow \infty} \frac{m(n, x)}{n}=\frac{6 \log 2 \log 10}{\pi^{2}} .
$$

Lochs' proof was based on the intricate arithmetic properties of the RCF map, and on a result by Lévy $[\mathrm{B}]$ which states that

$$
\lim _{n \rightarrow \infty} \frac{\log Q_{n}}{n}=\frac{\pi^{2}}{12 \log 2} \text { a.e. }(\lambda)
$$

where $\frac{P_{n}}{Q_{n}}=\left[0 ; a_{1}, a_{2}, \cdots, a_{n}\right]$.

In 1999, Bosma, Dajani and Kraaikamp [BDK] generalized Theorem 1 to a wider class of transformations on $[0,1)$ by noticing that Lochs's theorem is concerned with the way the decimal cylinders fit in the CF cylinders, and that the limit is in fact the ratio of the entropies of the maps under consideration. Their proof was based on the Shannon-McMillan-Breiman Theorem [ $[\vec{B}]$, and the dynamics of the underlying transformations as reflected in the way the partitions are refined under iterations of the corresponding maps.

For completeness, we state here the Shannon-McMillan-Breiman Theorem in the form in which we will use it.

Theorem 2 (Shannon-McMillan-Breiman). Let $T$ be an ergodic measure preserving transformation on a probability space $(X, \mathcal{B}, \mu)$, and let $P$ be a finite or countable generating partition for $T$ for which $H_{\mu}(P)<\infty$. Then for $\mu$-a.e. $x$,

$$
\lim _{n \rightarrow \infty} \frac{-\log \mu\left(P_{n}(x)\right)}{n}=h_{\mu}(T) .
$$

Here $H_{\mu}(P)$ denotes the entropy of the partition $P, h_{\mu}(T)$ denotes the entropy of $T$ and $P_{n}(x)$ denotes the element of the partition $\bigvee_{i=0}^{n-1} T^{-i} P$ containing $x$.

A surjective map $T:[0,1) \rightarrow[0,1)$ is called a number-theoretic fibered map (NTFM) if it satisfies the following conditions:

(a) there exists a finite or countable partition of intervals $\mathcal{P}=\left\{P_{i}: i \in D\right\}$ such that $T$ restricted to each atom of $\mathcal{P}$ (cylinder set of order 0 ) is monotone, continuous and injective,

(b) $T$ is ergodic with respect to Lebesgue measure $\lambda$, and there exists a $T$-invariant probability measure $\mu$ equivalent to $\lambda$ with bounded density. (Both $\frac{d \mu}{d \lambda}$ and $\frac{d \lambda}{d \mu}$ are bounded.)

Iterations of $T$ generate expansions of points $x \in[0,1)$ with digits in $D$. We refer to the resulting expansion as the $T$-expansion of $x$.

Almost all known expansions on $[0,1)$ are generated by a NTFM. Among them are the $n$-adic expansions $(T x=n x(\bmod 1)$, where $n$ is a positive integer $), \beta$ expansions $(T x=\beta x(\bmod 1)$, where $\beta>1$ is a real number $)$, continued fraction expansions $\left(T x=\frac{1}{x}-\left\lfloor\frac{1}{x}\right\rfloor\right)$, Lüroth series $\left(T x=\left\lfloor\frac{1}{x}\right\rfloor\left(\left\lfloor\frac{1}{x}\right\rfloor+1\right) x-\left\lfloor\frac{1}{x}\right\rfloor\right)$, and all $f$-expansions [R].

In [BDK], the authors call a NTFM $T r$-regular if, loosely speaking (i) the lengths of two adjacent $T$ cylinders of order $n$ (contained in the same $T$ cylinder of order $n-1$ ) differ by at most a constant factor $L$, and (ii) for any interval $I$ of $[0,1$ ), if 
$D_{m}$ is the smallest $T$ cylinder of order $m$ containing $I$, then for a.e. $x \in I, D_{m}$ has a refinement of order $m+r$ contained in $I$ with the property that it or an adjacent cylinder contains $x$. They show that the continued fraction map is 3-regular, and exhibit many examples with this property. They prove

Theorem 3 ([BDK]). Let $S$ and $T$ be NTFM's on $[0,1)$, and suppose $T$ is $r$ regular. Then for $\lambda$-a.e. $x$,

$$
\lim _{n \rightarrow \infty} \frac{m(n, x)}{n}=\frac{h(S)}{h(T)},
$$

where $h(S)$ and $h(T)$ denote the entropies of the maps $S$ and $T$ respectively, and $m(n, x)$ is the order of the smallest $T$ cylinder containing the $S$ cylinder of $x$ of order $n$.

Although $n$-adic expansions are not $r$-regular they were able to extend their result to the case when $S x=m x(\bmod 1)$ and $T x=n x(\bmod 1)$ indicating that $r$-regularity may not be needed. In this paper we show that Lochs' theorem is true for any two sequences of interval partitions on $[0,1)$ satisfying the conclusion of the Shannon-McMillan-Breiman theorem. As a corollary we prove a conjecture in BDK] that Loch's theorem is true for all pairs of number-theoretic fibered maps for which the generating partition has finite entropy.

We end this section with a few definitions that will be used in the arguments to follow.

Definition 1. By an interval partition, we mean a finite or countable partition of $[0,1)$ into subintervals. If $P$ is an interval partition and $x \in[0,1)$, we let $P(x)$ denote the interval of $P$ containing $x$.

Let $\mathcal{P}=\left\{P_{n}\right\}_{n=1}^{\infty}$ be a sequence of interval partitions. Let $\lambda$ denote a Borel probability measure on $[0,1)$.

Definition 2. Let $c \geq 0$. We say that $\mathcal{P}$ has entropy $c$ a.e. with respect to $\lambda$ if

$$
-\frac{\log \lambda\left(P_{n}(x)\right)}{n} \rightarrow c \text { a.e. }(\lambda) \text {. }
$$

We say that $\mathcal{P}$ has entropy $c$ in measure with respect to $\lambda$ if

$$
-\frac{\log \lambda\left(P_{n}(x)\right)}{n} \rightarrow c \text { in measure }(\lambda) .
$$

Note that we do not assume that each $P_{n}$ is refined by $P_{n+1}$.

Suppose that $\mathcal{P}=\left\{P_{n}\right\}_{n=1}^{\infty}$ and $\mathcal{Q}=\left\{Q_{n}\right\}_{n=1}^{\infty}$ are sequences of interval partitions. For each $n \in \mathbb{N}$ and $x \in[0,1)$, define

$$
m_{\mathcal{P}, \mathcal{Q}}(n, x)=\sup \left\{m \mid P_{n}(x) \subset Q_{m}(x)\right\} .
$$

\section{Almost everywhere convergence}

Theorem 4. Let $\mathcal{P}=\left\{P_{n}\right\}_{n=1}^{\infty}$ and $\mathcal{Q}=\left\{Q_{n}\right\}_{n=1}^{\infty}$ be sequences of interval partitions, and let $\lambda$ be a Borel probability measure on $[0,1)$. Suppose that for some constants $c>0$ and $d>0, \mathcal{P}$ has entropy $c$ a.e with respect to $\lambda$ and $\mathcal{Q}$ has entropy $d$ a.e. with respect to $\lambda$. Then

$$
\frac{m_{\mathcal{P}, \mathcal{Q}}(n, x)}{n} \rightarrow \frac{c}{d} \text { a.e. }(\lambda) .
$$


Proof. First we show that

$$
\limsup _{n \rightarrow \infty} \frac{m_{\mathcal{P}, \mathcal{Q}}(n, x)}{n} \leq \frac{c}{d} \text { a.e. }
$$

Fix $\varepsilon>0$. Let $x \in[0,1)$ be a point at which the convergence conditions of the hypotheses are met. Fix $\eta>0$ so that $\frac{c+\eta}{c-\frac{c}{d} \eta}<1+\varepsilon$. Choose $N$ so that for all $n \geq N$

$$
\lambda\left(P_{n}(x)\right)>2^{-n(c+\eta)}
$$

and

$$
\lambda\left(Q_{n}(x)\right)<2^{-n(d-\eta)} .
$$

Fix $n$ so that $\min \left\{n, \frac{c}{d} n\right\} \geq N$, and let $m^{\prime}$ denote any integer greater than $(1+\varepsilon) \frac{c}{d} n$. By the choice of $\eta$,

$$
\lambda\left(P_{n}(x)\right)>\lambda\left(Q_{m^{\prime}}(x)\right)
$$

so that $P_{n}(x)$ is not contained in $Q_{m^{\prime}}(x)$. Therefore

$$
m_{\mathcal{P}, \mathcal{Q}}(n, x) \leq(1+\varepsilon) \frac{c}{d} n
$$

and so

$$
\limsup _{n \rightarrow \infty} \frac{m_{\mathcal{P}, \mathcal{Q}}(n, x)}{n} \leq(1+\varepsilon) \frac{c}{d} \text { a.e. }
$$

Since $\varepsilon>0$ was arbitrary, we have the desired result.

Now we show that

$$
\liminf _{n \rightarrow \infty} \frac{m_{\mathcal{P}, \mathcal{Q}}(n, x)}{n} \geq \frac{c}{d} \text { a.e. }
$$

Fix $\varepsilon \in(0,1)$. Choose $\eta>0$ so that $\zeta=: \varepsilon c-\eta\left(1+(1-\varepsilon) \frac{c}{d}\right)>0$. For each $n \in \mathbb{N}$ let $\bar{m}(n)=\left\lfloor(1-\varepsilon) \frac{c}{d} n\right\rfloor$. For brevity, for each $n \in \mathbb{N}$ we call an element of $P_{n}$ (respectively $\left.Q_{n}\right)(n, \eta)$-good if

$$
\lambda\left(P_{n}(x)\right)<2^{-n(c-\eta)}
$$

(respectively

$$
\left.\lambda\left(Q_{n}(x)\right)>2^{-n(d+\eta)}\right) .
$$

For each $n \in \mathbb{N}$, let

$$
D_{n}(\eta)=\left\{x \mid \begin{array}{c}
P_{n}(x) \text { is }(n, \eta)-\operatorname{good} \text { and } Q_{\bar{m}(n)}(x) \text { is }(\bar{m}(n), \eta)-\operatorname{good} \\
\text { and } P_{n}(x) \nsubseteq Q_{\bar{m}(n)}(x)
\end{array}\right\} \text {. }
$$

If $x \in D_{n}(\eta)$, then $P_{n}(x)$ contains an endpoint of the $(\bar{m}(n), \eta)$-good interval $Q_{\bar{m}(n)}(x)$. By the definition of $D_{n}(\eta)$ and $\bar{m}(n)$,

$$
\frac{\lambda\left(P_{n}(x)\right)}{\lambda\left(Q_{\bar{m}(n)}(x)\right)} \leq 2^{-n \zeta} .
$$

Since no more than one atom of $P_{n}$ can contain a particular endpoint of an atom of $Q_{\bar{m}(n)}$, we see that $\lambda\left(D_{n}(\eta)\right) \leq 2 \cdot 2^{-n \zeta}$ and so

$$
\sum_{n=1}^{\infty} \lambda\left(D_{n}(\eta)\right)<\infty
$$


which implies that

$$
\lambda\left\{x \mid x \in D_{n}(\eta) \text { i.o. }\right\}=0 .
$$

Since $\bar{m}(n)$ goes to infinity as $n$ does, we have shown that for almost every $x \in[0,1)$, there exists $N \in \mathbb{N}$, so that for all $n \geq N, P_{n}(x)$ is $(n, \eta)$-good and $Q_{\bar{m}(n)}(x)$ is $(\bar{m}(n), \eta)$-good and $x \notin D_{n}(\eta)$. In other words, for almost every $x \in[0,1)$, there exists $N \in \mathbb{N}$, so that for all $n \geq N, P_{n}(x)$ is $(n, \eta)$-good and $Q_{\bar{m}(n)}(x)$ is $(\bar{m}(n), \eta)$ good and $P_{n}(x) \subset Q_{\bar{m}(n)}(x)$. Thus, for almost every $x \in[0,1)$, there exists $N \in \mathbb{N}$, so that for all $n \geq N, m_{\mathcal{P}, \mathcal{Q}}(n, x) \geq \bar{m}(n)$, so that

$$
\frac{m_{\mathcal{P}, \mathcal{Q}}(n, x)}{n} \geq \frac{\left\lfloor(1-\varepsilon) \frac{c}{d} n\right\rfloor}{n} .
$$

This proves that

$$
\liminf _{n \rightarrow \infty} \frac{m_{\mathcal{P}, \mathcal{Q}}(n, x)}{n} \geq(1-\varepsilon) \frac{c}{d} \text { a.e. }
$$

Since $\varepsilon>0$ was arbitrary, we have established the theorem.

\section{Application}

Let $S$ and $T$ be number-theoretic fibered maps on $[0,1)$ with invariant probability measures $\mu_{1}$ and $\mu_{2}$, respectively, each boundedly equivalent to Lebesgue measure and with generating partitions (cylinders of order 0) $P$ and $Q$ respectively. Denote by $P_{n}$ and $Q_{n}$ the interval partitions of $[0,1)$ into cylinder sets of order $n$, and, as above, denote by $P_{n}(x)$ the element of $P_{n}$ containing $x$ (similarly for $Q_{n}(x)$ ), and set

$$
m(n, x)=\sup \left\{m \mid P_{n}(x) \subset Q_{m}(x)\right\} .
$$

Suppose that $H_{\mu_{1}}(P)$ and $H_{\mu_{2}}(Q)$ are finite and $h_{\mu_{1}}(S)$ and $h_{\mu_{2}}(T)$ are positive.

Theorem 5. Let $S$ and $T$ be as above and let $\lambda$ denote Lebesgue measure on $[0,1)$. Then

$$
\lim _{n \rightarrow \infty} \frac{m(n, x)}{n}=\frac{h(S)}{h(T)} \text { a.e. }(\lambda) .
$$

Proof. Since $\left(S, \mu_{1}\right)$ and $\left(T, \mu_{2}\right)$ are ergodic, by the Shannon-McMillan-Breiman theorem,

$$
-\frac{\log \mu_{1}\left(P_{n}(x)\right)}{n} \rightarrow h_{\mu_{1}}(S) \text { a.e. }\left(\mu_{1}\right)
$$

and

$$
-\frac{\log \mu_{2}\left(Q_{n}(x)\right)}{n} \rightarrow h_{\mu_{2}}(T) \text { a.e. }\left(\mu_{2}\right),
$$

where $h(S)>0$ and $h(T)>0$. Since $\mu_{1}$ and $\mu_{2}$ are equivalent to $\lambda$ and have bounded densities with respect to $\lambda$, it follows that in the above limits one can replace $\mu_{1}$ and $\mu_{2}$ by $\lambda$. By Theorem 4 the result follows. 


\section{Convergence in measure}

In general, a sequence of interval partitions $\mathcal{P}=\left\{P_{n}\right\}_{n=1}^{\infty}$ may have entropy in measure with respect to a measure $\lambda$, but no entropy a.e. $(\lambda)$. However, given a pair of such sequences of partitions, and with one additional hypothesis, we obtain a convergence in measure result analogous to the previous theorem.

Theorem 6. Let $\mathcal{P}=\left\{P_{n}\right\}_{n=1}^{\infty}$ and $\mathcal{Q}=\left\{Q_{n}\right\}_{n=1}^{\infty}$ be sequences of interval partitions, and let $\lambda$ be a Borel probability measure on $[0,1)$. Suppose that for some constants $c>0$ and $d>0, \mathcal{P}$ has entropy $c$ in measure and $\mathcal{Q}$ has entropy $d$ in measure. Suppose in addition that for each $n, Q_{n}$ is refined by $Q_{n+1}$. Then

$$
\frac{m_{\mathcal{P}, \mathcal{Q}}(n, x)}{n} \rightarrow \frac{c}{d} \text { in measure. }
$$

Proof. Fix $\varepsilon \in(0,1)$. Choose $\eta>0$ so that $\eta<\varepsilon$ and $\zeta=:(c-\eta)-(1-\varepsilon)\left(c+\frac{c}{d} \eta\right)$ $>0$. Choose $N$ so large that for all $n \geq N$,

$$
\lambda\left\{x||-\frac{\log \lambda\left(P_{n}(x)\right)}{n}-c \mid<\eta\right\}>1-\eta
$$

and

$$
\lambda\left\{x||-\frac{\log \lambda\left(Q_{n}(x)\right)}{n}-d \mid<\eta\right\}>1-\eta .
$$

We refer to a partition element $P_{n}(x)$ as $(n, \eta)$-good if it satisfies

$$
\left|-\frac{\log \lambda\left(P_{n}(x)\right)}{n}-c\right|<\eta
$$

and make a similar definition for $Q_{n}(x)$. Choose $n>N$ so that $m(n)=:\left\lfloor(1-\varepsilon) \frac{c}{d} n\right\rfloor$ $>N$ and $2^{-n \zeta}<\varepsilon$. Each $(n, \eta)-\operatorname{good} P_{n}(x)$ and $(m(n), \eta)-\operatorname{good} Q_{m}(y)$ satisfy

$$
\lambda\left(P_{n}(x)\right)<2^{-n(c-\eta)}
$$

and

$$
\lambda\left(Q_{m}(y)\right)>2^{-m(n)(d+\eta)}
$$

so that

$$
\frac{\lambda\left(P_{n}(x)\right)}{\lambda\left(Q_{m}(y)\right)}<\frac{2^{-n(c-\eta)}}{2^{-m(n)(d+\eta)}} \leq 2^{-n \zeta}<\varepsilon .
$$

Consider the set $B_{n}=\left\{x \in[0,1) \mid P_{n}(x) \nsubseteq Q_{m(n)}(x)\right\}$. We have

$$
\begin{aligned}
B_{n} & \subset\left\{x \mid P_{n}(x) \text { is not }(n, \eta) \text {-good }\right\} \\
& \cup\left\{x \mid P_{n}(x) \text { is disjoint from all }(m(n), \eta) \text {-good } Q_{m(n)}(y)\right\} \\
& \cup\left\{x \mid \begin{array}{r}
P_{n}(x) \text { is }(n, \eta) \text {-good, } P_{n}(x) \text { meets some }(m(n), \eta) \text {-good } \\
Q_{m(n)}(y), \text { but } P_{n}(x) \text { is not contained in } Q_{m(n)}(x)
\end{array}\right\} .
\end{aligned}
$$

Each of the first two of these sets has measure less than $\eta$. If $x$ is in the third set, then $P_{n}(x)$ contains an endpoint of some $(m(n), \eta)$-good $Q_{m(n)}(y)$. No other element of the partition $P_{n}$ could contain that endpoint. Thus there can be at most two such $P_{n}$ elements for each $(m, \eta)$-good $Q_{m}$ element. Because of the ratio of the lengths of $(n, \eta)$-good $P_{n}$ and $(m, \eta)$-good $Q_{m}$ elements, we see that the measure of the third set cannot exceed $2 \varepsilon$. Thus $\lambda\left(B_{n}\right)<2 \varepsilon+2 \eta<4 \varepsilon$. 
If $x \in[0,1] \backslash B_{n}$, then $m_{\mathcal{P}, \mathcal{Q}}(n, x) \geq m(n)$, so

$$
\frac{m_{\mathcal{P}, \mathcal{Q}}(n, x)}{n} \geq \frac{\left\lfloor(1-\varepsilon) \frac{c}{d} n\right\rfloor}{n} .
$$

Since $\varepsilon$ was arbitrary, we have shown that for all $\delta>0$ there is an $N$ so that for all $n>N$,

$$
\lambda\left\{x \mid \frac{m_{\mathcal{P}, \mathcal{Q}}(n, x)}{n}>(1-\delta) \frac{c}{d}\right\} \geq 1-\delta .
$$

On the other hand, given $\varepsilon>0$, suppose $\eta>0$ is chosen so that $(1+\varepsilon)\left(c-\frac{c}{d} \eta\right)-$ $(c+\eta)>0$ and $N$ is chosen as before. Let $n \geq N$ and let $m^{\prime}$ denote any integer greater than $(1+\varepsilon) \frac{c}{d} n$. Then each $(n, \eta)$-good element of $P_{n}$ has measure greater than the measure of each $\left(m^{\prime}, \eta\right)$-good element of $Q_{m^{\prime}}$. Therefore

$$
\begin{aligned}
C_{n}=: & \left\{x \mid P_{n}(x) \subset Q_{m^{\prime}}(x)\right\} \\
\subset\left\{x \mid P_{n}(x) \text { is not }(n, \eta) \text {-good }\right\} & \\
& \cup\left\{x \mid Q_{m^{\prime}}(x) \text { is not }\left(m^{\prime}, \eta\right) \text {-good }\right\}
\end{aligned}
$$

so that $\lambda\left\{x \mid P_{n}(x) \subset Q_{m^{\prime}}(x)\right\} \leq 2 \eta$. But for all $m>m^{\prime}, Q_{m^{\prime}}$ is refined by $Q_{m}$, so that $Q_{m}(x) \subset Q_{m^{\prime}}(x)$. Thus if $x \in[0,1) \backslash C_{n}$ and $m>m^{\prime}$, then $P_{n}(x) \nsubseteq Q_{m}(x)$. In other words, for each $x \in[0,1] \backslash C_{n}, m_{\mathcal{P}, \mathcal{Q}}(n, x)<m^{\prime}$ so that

$$
\frac{m_{\mathcal{P}, \mathcal{Q}}(n, x)}{n} \leq \frac{(1+\varepsilon) \frac{c}{d} n}{n} \text {. }
$$

Since $\varepsilon$ was arbitrary, we have shown that for all $\delta>0$ there is an $N$ so that for all $n>N$,

$$
\lambda\left\{x \mid \frac{m_{\mathcal{P}, \mathcal{Q}}(n, x)}{n}<(1+\delta) \frac{c}{d}\right\} \geq 1-\delta,
$$

which completes the proof.

Remark 1. The above results (and hence the application) can be extended to sequences of interval partitions $\mathcal{P}=\left\{P_{n}\right\}_{n=1}^{\infty}$ and $\mathcal{Q}=\left\{Q_{n}\right\}_{n=1}^{\infty}$ of entropy $c$ and $d$, where exactly one of the numbers $c$ and $d$ is zero. If $c=0$ and $d \neq 0$, we can conclude that

$$
\lim _{n \rightarrow \infty} \frac{m_{\mathcal{P}, \mathcal{Q}}(n, x)}{n}=0 .
$$

In the reverse case we conclude

$$
\lim _{n \rightarrow \infty} \frac{m_{\mathcal{P}, \mathcal{Q}}(n, x)}{n}=\infty .
$$

The proofs are easy adaptations of the ones given.

\section{REFERENCES}

[BBDK] Barrionuevo, J., R.M. Burton, K. Dajani and C. Kraaikamp - Ergodic properties of generalized Lúroth series, Acta Arithm., 74 (1996), 311-327. MR 97b:11104

[BDK] Bosma, W., K. Dajani and C. Kraaikamp - Entropy and counting correct digits, University of Nijmegen, report no. 9925, 1999. http://www-math.sci.kun.nl /math/onderzoek/reports/reports1999.html

[B] Billingsley, P. - Ergodic Theory and Information, John Wiley and Sons, Inc.; New YorkLondon-Sydney (1965). MR 33:254

[L] Lochs, G. - Vergleich der Genauigkeit von Dezimalbruch und Kettenbruch, Abh. Math. Sem. Hamburg, 27 (1964), 142-144. MR 29:57 
[R] Rényi, A. - Representations for real numbers and their ergodic properties, Acta Math. Acad. Sci. Hung. 8 (1957), 472-493. MR 20:3843

[S] Schweiger, F. - Ergodic Theory of Fibred Systems and Metric Number Theory, Oxford University Press, Oxford, 1995. MR 97h:11083

Department of Mathematics, Utrecht University, 3508TA Utrecht, The Netherlands E-mail address: dajani@math.uu.nl

Department of Mathematics, Wesleyan University, Middletown, Connecticut 06459

E-mail address: afieldsteel@wesleyan.edu 